\title{
On the Maximum Sum-Rate of Cognitive MIMO Interference Channels
}

\author{
Harpreet S. Dhillon and R. Michael Buehrer \\ Mobile and Portable Radio Research Group (MPRG), \\ Wireless@ Virginia Tech, Bradley Department of Electrical and Computer Engineering, \\ Virginia Polytechnic Institute and State University, Blacksburg, VA 24061, USA. \\ E-mail: \{harpreet.dhillon, buehrer\}@vt.edu
}

\begin{abstract}
In this paper, we address the problem of maximizing the ergodic sum-rate of an $N$-user cognitive MIMO Interference Channel (IC) formed by unlicensed (or secondary) users. We assume that $N$ secondary users coexist in the same area and try to access the same set of frequency bands. In such a setup, we define a cognitive MIMO IC as an extension of a classical IC where each user has multiple antennas and multiple frequency bands for transmission. We further assume a typical cognitive radio scenario where the structure of interference signals is not known and interference cancelation is not possible. Therefore, interference is treated as Gaussian noise in this work. The problem of ergodic sum-rate maximization reduces to the problem of finding the optimal power allocation for each user over each spatial channel in each frequency band. This problem belongs to the class of non-linear non-convex optimization problems and is hence challenging to solve analytically. We extend a reformulation and linearization (RLT) based branch and bound (BB) method to solve this problem. BB/RLT was recently proposed to solve the sum-rate maximization problem of a single band MIMO IC with the guaranteed convergence to the global optimum. It should be noted that we are proposing an optimal power control scheme to perform power control simultaneously over spatial and spectral dimensions without any explicit interference cancelation or Interference Alignment (IA). We also present some interesting comparisons between the sum-rates achievable by the proposed scheme and those by IA.
\end{abstract}

Index Terms-Cognitive MIMO radio, interference channels, interference alignment, maximum sum-rate, non-linear nonconvex programming.

\section{INTRODUCTION}

$\mathbf{C}$ OGNITIVE radio has been one of the most researched topics of the last decade. It has attracted the attention of industry and academia alike. The concept of 'cognitive radio' (CR) was introduced by Mitola in late 1990s as an enhancement on the traditional software radio concept [1]-[2]. The key idea of $\mathrm{CR}$ is to add cognition to radios so that they can adapt to the changing electromagnetic environment to maximize the utilization of resources (especially spectrum) in the network [3]-[4]. The interest in CR was further fueled by the Federal Communications Commission (FCC) measurements that clearly indicated the under utilization of the spectrum in the existing fixed spectrum allocation regime [5]. In the fixed spectrum regime, each wireless user/technology is allocated a fixed frequency band that can not be utilized by other technologies. Though this type of spectrum allocation avoids interference, it is not optimal from the spectral utilization view point, as shown by [5]. The inefficiency of fixed spectrum allocation has led to a plenty of research activities around the dynamic spectrum access aspect of CR [4], [6].

Dynamic spectrum access models can be categorized into two main groups, viz., open sharing models and hierarchical models. Open sharing models consider all the users as equals and there is no concept of licensing the spectrum [8]. On the other hand, hierarchical models consider that the unlicensed 'cognitive' (or secondary) users share the spectrum with the licensed (or primary) users. The basic idea is to allow the secondary users (SUs) to access the licensed spectrum while keeping the interference perceived by the primary users (PUs) within limits [6]. Two major spectrum access strategies within hierarchical models are spectrum underlay and spectrum overlay. The underlay approach allows the SUs to access the licensed spectrum with the condition that they will operate below the noise floor of PUs. On the other hand, the overlay approach allows the SUs to transmit only on those frequency bands where PUs are not transmitting. In this paper, we consider hierarchical access model with the spectrum overlay approach for dynamic spectrum access. This approach is also referred to as opportunistic dynamic spectrum access [6]. The main steps involved in this approach are to first identify the available frequency bands (white spaces) in the spectrum and then allocate them to the SUs. In this paper, we assume that we have perfect knowledge of the white spaces and will primarily focus on dynamic spectrum allocation (DSA) aspect. Interested readers can refer to [9] for a discussion on various spectrum sensing techniques.

In this work, we assume that each SU has multiple antennas both at the transmitter ( $\mathrm{Tx})$ and receiver $(\mathrm{Rx})$ nodes. The main goal of this work is to develop an optimal spatiospectral power control technique to maximize the ergodic sumrate of these MIMO SUs treating interference as Gaussian noise. Since all the SUs mutually interfere, this network can be analyzed as an extension of a classical MIMO IC with the addition that each SU has multiple frequency bands for transmission. It is worth noting that the characterization of the capacity region of even the classical single-band IC is a long standing open problem. It was introduced by Shannon in early 1960s [10]. Our understanding of the IC capacity region is severely limited despite persistent research efforts spanned over several decades [11]-[13]. Until recently, we just had some insight into a few special cases of the simple two- 
user Gaussian IC, such as the very high interference case [14][15]. In an important recent step, the capacity of a two-user Gaussian IC was characterized to within one-bit of the true channel capacity [16].

To work around this problem, some researchers have taken an alternate approach and mapped this capacity characterization problem to the problem of characterizing the degrees of freedom of an IC [17], [18]. An IC is said to have $D$ degrees of freedom, if the sum-rate $(S R)$ can be expressed as:

$$
S R=D \log (S N R)+o(\log (S N R)),
$$

where $\log (S N R)$ represents the capacity of an isolated user, $o(\log (S N R))$ is the residual capacity term and $S N R$ is the signal-to-noise ratio. The most remarkable result of these studies is that an $N$-user IC has $N / 2$ degrees of freedom per orthogonal spectral, spatial or temporal slots and can thus achieve at least half the sum-rate of its no-interference counterpart for any number of interferers. It has been further shown that these $N / 2$ degrees of freedom are achievable by Interference Alignment (IA) [18]. The idea of IA is to precode the transmit symbols such that they are orthogonal to the interference at the intended Rx node and aligns with the other interference signals at the rest of Rx nodes. Several precoding methods are shown to achieve IA for single antenna ICs with time or frequency selectivity [19], [20]. $N$-user MIMO interference channels are also shown to achieve $N / 2$ degrees of freedom when the channels have infinite selectivity [21]. However, it is difficult to find analytical solutions to the IA problems in general and even the feasibility of IA over a limited number of signaling dimensions is an open problem [22].

Another popular way of characterizing the capacity of an IC is to maximize the sum-rate by treating interference as Gaussian noise. The cognitive MIMO sum-rate maximization problem was first formulated in [23] by extending the sumrate maximization problem of a single-band MIMO IC [24]. These optimization problems are known to be non-linear nonconvex and are hence difficult to solve analytically. Even the popular local optimization algorithms, such as gradient based search, can not guarantee global optimal solutions for nonconvex problems. Recently, a global optimization algorithm was developed in [25] by coupling the reformulation and linearization technique (RLT) with the Branch and Bound (BB) strategy to find the global optimal sum-rate of a single-band MIMO IC under the assumption that each user distributes its transmit power equally over all the spatial channels. This assumption was relaxed in [26] to find the optimal sum-rate of the general single-band MIMO IC. In this paper, we extend the BB/RLT algorithm to find the maximum achievable sumrate of the cognitive MIMO IC. It is important to note here that this problem is essentially a power control problem where we control the transmit power simultaneously over spectral and spatial domains subject to a maximum power constraint. IA in not explicitly considered in our setup. However, we compare the sum-rate achievable by optimal power control with the one achievable by IA and show that power control performs significantly better in the low interference and low $S N R$ regimes. This result is significant from the $\mathrm{CR}$ perspective where the SUs mostly operate in the low $S N R$ regimes. We further show that the presence of more orthogonal spatial or spectral channels improves the performance of power control as compared to IA.

The remainder of the paper is organized as follows. The system model is introduced in Section II. Sum-rate maximization problem of cognitive MIMO ICs is discussed in Section III. The proposed BB/RLT global optimization algorithm is presented in Section IV. Section V deals with the numerical results and the paper is concluded in Section VI.

\section{SySTEM MOdEL}

\section{A. Assumptions}

Several assumptions are made in the analysis to facilitate the system layout. Firstly, it is assumed that we have perfect knowledge of the white spaces. Thus, we assume that no PU is present in the frequency bands of interest. Secondly, we assume that all the SUs consist of a transmitter (Tx) comprising of $n_{t}$ transmit antennas and a receiver (Rx) comprising of $n_{r}$ receive antennas. In this MIMO IC model, each Tx has only one intended $\mathrm{Rx}$ and acts as an interferer for the rest of the SUs. Further, we make the Gaussian interference channel assumption in which interference and noise are modeled as being Gaussian distributed. Thirdly, we assume that the available spectrum is divided into a countable number of orthogonal frequency bands. SUs are not restricted to transmit over a single band and can distribute transmit power over multiple frequency bands. Each $\mathrm{Tx}$ is assumed to have a total maximum transmit power of $P_{\max }$ over all frequency bands and all $n_{t}$ transmit antennas. It is further assumed that all the users are sharing complete information and the system is centrally optimized to find the maximum sum of the mutual information. In this case, a centralized server obtains information about the topology (e.g., channel gains) and determines the optimal spatio-spectral power allocation for all the SUs.

\section{B. System Layout}

The system layout is shown in Fig. 1. The maximum allowable distance between a Tx/Rx pair is denoted by $d_{\max }$. $d_{\max }$ is assumed to be a system constant and is defined such that the minimum average received SNR per receive antenna is $S N R_{\min } \mathrm{dB}$ (assuming no fading or shadowing). The density of the users is handled by defining a Multi-User Interference $(M U I)$ factor. $M U I$ represents the expected number of $\mathrm{Rx}$ nodes within a circle of radius $d_{\max }$ centered at any $\mathrm{Tx}$ node assuming a constant density. Increasing the $M U I$ factor increases the density of interferers and hence increases the mutual interference. For a fixed $M U I$ and $d_{\max }$ value, the density $(\mu)$ in terms of users per unit area can be evaluated as: $\mu=M U I / \pi d_{\max }^{2}$. The complete area of interest is assumed to be a square. To place $N$ users within a square with density $\mu$, the square should have an area of $\frac{N}{\mu}$, and hence a side length of $\sqrt{\frac{N}{\mu}}$. For each analysis, we place a number of Rx units uniformly in the chosen square area. Each Tx is then placed in the circle of radius $d_{\max }$ centered at the corresponding $\mathrm{Rx}$, as shown in Fig. 1. We simulate this system a number of times 


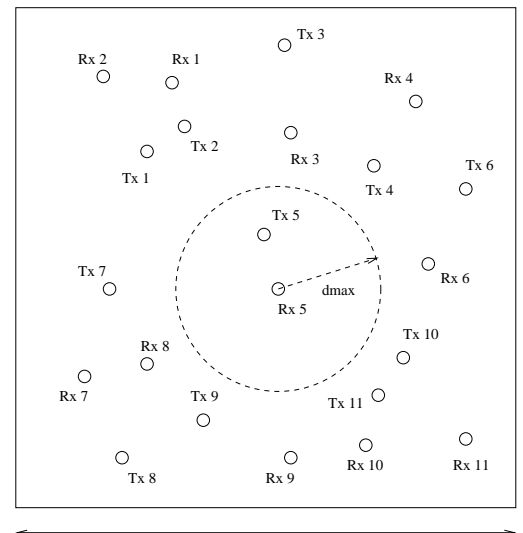

Side Length $(\mathrm{L})=(\mathrm{N} / \mu)^{1 / 2}$

Fig. 1. System model depicting various Tx-Rx pairs.

to find the ergodic sum-rate and for each simulation we place the users randomly as described above.

\section{Mathematical Notations}

Boldface is used to denote matrices and vectors. For a matrix $\mathbf{A}, \mathbf{A}^{\dagger}$ denotes the conjugate transpose and $\mathbf{A}^{T}$ denotes the transpose. $\operatorname{Tr}\{\mathbf{A}\}$ denotes the trace of the matrix $\mathbf{A}$. I denotes the identity matrix, whose dimensions can be determined from the context. $\mathbf{A} \succ \mathbf{0}$ represents that $\mathbf{A}$ is Hermitian and positive semidefinite. The scalar $a_{m, n}$ represents the entry in the $m^{t h}$ row and $n^{t h}$-column of $\mathbf{A}$. For a complex scalar $a, \Re(a)$ and $\Im(a)$ represent the real and imaginary parts of $a$, respectively, and $a^{\prime}$ represents the conjugate of $a . \operatorname{diag}\{\mathbf{A}\}$ denotes a vector of the diagonal elements of $\mathbf{A}$ and $\operatorname{diag}\{\mathbf{A}\} \succeq \mathbf{0}$ means that all the diagonal elements of $\mathbf{A}$ are non-negative.

\section{Sum-RAte of Cognitive MiMO IC}

In this section, we briefly formulate the sum-rate maximization problem of cognitive MIMO ICs. It should be noted that this problem was first formulated in [23] by incorporating DSA in multiuser MIMO networks.

\section{A. Defining the Variables}

We consider an IC consisting of $N$ mutually interfering SUs, which are indexed by $1,2, \ldots, N$. In this analysis, it is assumed that the transmitters have full channel state information. Let the available spectrum be divided into $m$ frequency bands, indexed by $1,2, \ldots, m$. Let us denote the MIMO link from the Tx of the $j^{t h} \mathrm{SU}$ to the $\mathrm{Rx}$ of the $i^{t h}$ SU to be $L_{j i}$. Let the matrix $\mathbf{H}_{j i}^{l} \in C^{n_{r} \times n_{t}}$ denote the channel matrix of link $L_{j i}$ in the $l^{t h}$ frequency band. Let the matrix $\mathbf{Q}_{i}^{l}$ be the covariance matrix of the zero-mean Gaussian transmit symbol vector $\mathbf{x}_{i}^{l}$ of the $i^{\text {th }} \mathrm{SU}$ in $l^{\text {th }}$ frequency band, i.e., $\mathbf{Q}_{i}^{l}=E\left\{\mathbf{x}_{i}^{l} \cdot \mathbf{x}_{i}^{l \dagger}\right\}$. Further denote $\rho_{j i}^{l}$ as the signal-to-noise ratio per unit transmit power in frequency band $l$ if $j=i$, or the interference-to-noise ratio per unit transmit power if $j \neq i$. It is also assumed that each $\mathrm{Tx}$ in the network is subject to the maximum transmit power constraint, i.e., the total power transmitted over $n_{t}$ transmit antennas and all $m$ frequency bands should be less than or equal to $P_{\max }$. Let
$\mathbf{R}_{i}^{l}$ represent the covariance matrix of the interference plus noise observed at the $i^{\text {th }} \mathrm{Rx}$ node in the $l^{\text {th }}$ frequency band. Assuming interference plus noise to be Gaussian distributed, it can be computed as:

$$
\mathbf{R}_{i}^{l}=\sum_{\substack{j=1 \\ j \neq i}}^{N} \rho_{j i}^{l} \mathbf{H}_{j i}^{l} \mathbf{Q}_{j}^{l} \mathbf{H}_{j i}^{l \dagger}+\mathbf{I} .
$$

\section{B. Sum-rate of Cognitive MIMO IC}

We begin by analyzing the capacity of a single MIMO link which can be computed as $C_{i}=\sum_{l=1}^{m} \log _{2} \operatorname{det}(\mathbf{I}+$ $\left.\rho_{i i}^{l}\left(\mathbf{R}_{i}^{l}\right)^{-1} \mathbf{H}_{i i}^{l} \mathbf{Q}_{i}^{l} \mathbf{H}_{i i}^{l \dagger}\right)$. Since $\mathbf{Q}_{i}^{l} \succ \mathbf{0}$, it can be expressed as $\mathbf{Q}_{i}^{l}=\mathbf{U}_{i}^{l} \Lambda_{i}^{l} \mathbf{U}_{i}^{l \dagger}$. Defining $\widehat{\mathbf{H}}_{i i}^{l}=\mathbf{H}_{i i}^{l} \mathbf{U}_{i}^{l}$, the capacity of the single MIMO link in this case can be written as $C_{i}=\sum_{l=1}^{m} \log _{2} \operatorname{det}\left(\mathbf{I}+\rho_{i i}^{l}\left(\mathbf{R}_{i}^{l}\right)^{-1} \widehat{\mathbf{H}}_{i i}^{l} \Lambda_{i}^{l} \widehat{\mathbf{H}}_{i i}^{l \dagger}\right)$. As $\mathbf{Q}_{i} \succ \mathbf{0}$, it leads to the following two very important properties which are instrumental in the further simplification of the problem formulation:

1) The distributions of $\widehat{\mathbf{H}}_{i i}^{l}$ and $\mathbf{H}_{i i}^{l}$ are same [24].

2) All the eigenvalues of $\mathbf{Q}_{i}^{l}$, i.e. all the diagonal elements of $\Lambda_{i}^{l}$, are real and positive.

Due to these properties, it is sufficient to consider $\Lambda_{i}$ instead of $\mathbf{Q}_{i}$ in the problem formulation. It should, however, be noted that the instantaneous sum-rate of the original and transformed formulation is not necessarily the same. It is only the ergodic sum-rate that remains the same. Using this information, the problem of cognitive MIMO ergodic sum-rate maximization can now be formulated as:

$$
\begin{array}{ll}
\max & \sum_{i=1}^{N} C_{i} \\
\text { s.t. } & C_{i}=\sum_{l=1}^{m} \log _{2} \operatorname{det}\left(\mathbf{I}+\rho_{i i}^{l} \mathbf{R}_{i}^{l-1} \widehat{\mathbf{H}}_{i i}^{l} \Lambda_{i}^{l} \widehat{\mathbf{H}}_{i i}^{l \dagger}\right) \\
& \mathbf{R}_{i}^{l}=\sum_{\substack{j=1 \\
j \neq i}}^{N} \rho_{j i} \widehat{\mathbf{H}}_{j i}^{l} \Lambda_{j}^{l} \widehat{\mathbf{H}}_{j i}^{l \dagger}+\mathbf{I} \\
& \sum_{l=1}^{m} \operatorname{Tr}\left\{\Lambda_{i}^{l}\right\} \leq P_{\max }, \operatorname{diag}\left\{\Lambda_{i}^{l}\right\} \succeq \mathbf{0} \\
& 1 \leq i \leq N, 1 \leq l \leq m .
\end{array}
$$

The above formulation basically finds the power transmitted by each user in each channel-mode in each frequency band that maximizes the sum-rate. It should be noted that the channelmodes of each user forming an IC are not necessarily the same as the channel-modes of its no-interference counterpart. This problem is non-linear non-convex and hence its global optimal solution can not be derived analytically. Even the popular local optimization algorithms, such as gradient based search do not guarantee global optimal solution. To overcome this problem, we now develop a BB/RLT based global optimization algorithm which has a guaranteed convergence to the global optimal solution.

\section{The BB/RLT Global Optimization Algorithm}

BB/RLT was proposed in [25], and later extended in [26], for solving the sum-rate maximization problem of the single band MIMO IC. We now extend this algorithm to encompass the cognitive MIMO IC case in the general formulation presented in [26]. It should be noted that the RLT would be different in this case because of the different problem formulation. However, the BB strategy would remain the same 
as that of [26] and is omitted here to save space. We begin our discussion by presenting a brief overview of BB/RLT.

\section{A. Brief Overview of $B B / R L T$}

The basic idea of BB/RLT is to first find the upper bound (UB) and the lower bound (LB) to the optimal sum-rate by constructing a linear programming relaxation (LPR) of the original non-linear (NL) problem and then tightening the bounds by employing the BB strategy [25], [26]. While constructing the LPR, all the NL terms in the objective function and the constraints are replaced by linear variables by introducing suitable linear constraints, thereby relaxing the feasible region. The optimal objective function value of this LP thus serves as an upper bound (UB) to the original NL problem (since it is a maximization problem). If the optimal solution of this relaxed LP is feasible to the original NL problem, it acts as a lower bound (LB) to the globally optimal value (since it is sub-optimal to the original NL problem in general). Otherwise, it can be used as a starting point to find a feasible solution by local search. After finding the UB and LB, the BB strategy is employed to partition the search space so that both the largest UB and LB approach the global optimum. When the largest UB and the LB are within some threshold, $\epsilon$, the BB/RLT is complete and yields a LB to the global optimum that is within $\epsilon$ of the true global optimum [25], [26].

Constructing an LPR: We begin by defining the following new variables to linearize the expression of $C_{i}$ :

$$
\begin{aligned}
& x_{i}^{l}=\operatorname{det}\left(\mathbf{R}_{i}^{l}+\rho_{i i}^{l} \widehat{\mathbf{H}}_{i i}^{l} \Lambda_{i}^{l} \widehat{\mathbf{H}}_{i i}^{l \dagger}\right) ; u_{i}^{l}=\ln \left(x_{i}^{l}\right) ; \\
& y_{i}^{l}=\operatorname{det}\left(\mathbf{R}_{i}^{l}\right) ; v_{i}^{l}=\ln \left(y_{i}^{l}\right) .
\end{aligned}
$$

$C_{i}$ can be expressed in terms of the new variables as: $C_{i}=$ $\sum_{l=1}^{m} \frac{1}{\ln 2}\left(u_{i}^{l}-v_{i}^{l}\right)$. The constraints given by (4) are added to the problem formulation given by (3). $u_{i}^{l}$ and $v_{i}^{l}$ are logarithmic functions of the form $w=\ln z$, where $z \in\left[z_{L B} z_{U B}\right]$. Since, $x_{i}^{l}$ and $y_{i}^{l}$ are dependent upon $\Lambda_{i}^{l}$, their LB and UB can be calculated by evaluating their expressions at the LB and UB of $\Lambda_{i}^{l}$ respectively. A convex polygonal outer approximation is used to linearize the logarithmic function [26]. As shown in Fig. 2, the convex region is defined by the tangents at $\left(z_{L B}, \ln z_{L B}\right),\left(z_{\gamma}, \ln z_{\gamma}\right)$ and $\left(z_{U B}, \ln z_{U B}\right)$, and the chord joining $\left(z_{L B}, \ln z_{L B}\right)$ and $\left(z_{U B}, \ln z_{U B}\right)$, where $z_{\gamma}$ is the $\mathrm{z}$-coordinate of the point of intersection of the tangents at $\left(z_{L B}, \ln z_{L B}\right)$ and $\left(z_{U B}, \ln z_{U B}\right)$, which is given by:

$$
z_{\gamma}=\frac{z_{L B} z_{U B}\left(\ln z_{U B}-\ln z_{L B}\right)}{z_{U B}-z_{L B}} .
$$

The convex region defined by these four segments can be expressed by the following linear constraints:

$$
\begin{aligned}
& z_{L B} w-z \leq z_{L B}\left(\ln z_{L B}-1\right), \\
& z_{\gamma} w-z \leq z_{\gamma}\left(\ln z_{\gamma}-1\right), \\
& z_{U B} w-z \leq z_{U B}\left(\ln z_{U B}-1\right), \\
& \left(z_{U B}-z_{L B}\right) w+\left(\ln z_{L B}-\ln z_{U B}\right) z \geq \\
& z_{U B} \ln z_{L B}-z_{L B} \ln z_{U B} .
\end{aligned}
$$

After linearizing $u_{i}^{l}$ and $v_{i}^{l}$, we linearize $x_{i}^{l}$ and $y_{i}^{l}$. The expressions for $x_{i}^{l}$ and $y_{i}^{l}$ are quite similar and this similarity

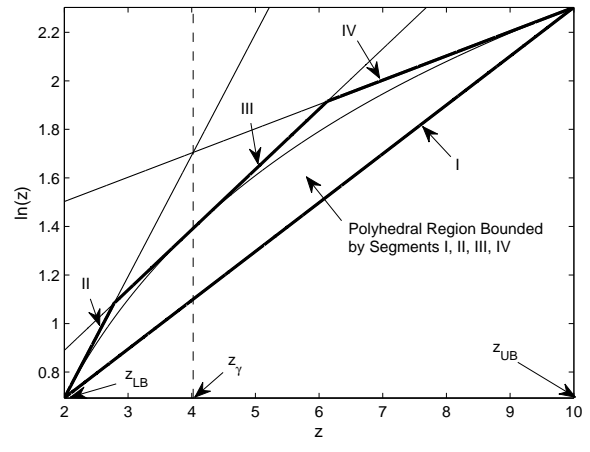

Fig. 2. Polyhedral approximation of $w=\ln z$.

can be established by substituting $\mathbf{R}_{i}^{l}$ in (4):

$$
\begin{aligned}
& x_{i}^{l}=\operatorname{det}\left(\sum_{j=1}^{N}\left(\rho_{j i}^{l} \widehat{\mathbf{H}}_{j i}^{l} \Lambda_{j}^{l} \widehat{\mathbf{H}}_{j i}^{l \dagger}+\mathbf{I}\right)\right) \triangleq \operatorname{det} \mathbf{S}_{i}^{l}, \\
& y_{i}^{l}=\operatorname{det}\left(\sum_{\substack{j=1 \\
j \neq i}}^{N}\left(\rho_{j i}^{l} \widehat{\mathbf{H}}_{j i}^{l} \Lambda_{j}^{l} \widehat{\mathbf{H}}_{j i}^{l \dagger}+\mathbf{I}\right)\right) \triangleq \operatorname{det} \mathbf{R}_{i}^{l} .
\end{aligned}
$$

Keeping in mind this similarity, we illustrate the linearization process for only $x_{i}^{l}$, and the results for $y_{i}^{l}$ can be inferred directly. For the purpose of illustration, we assume that Tx and $\mathrm{Rx}$ nodes of each user have two antennas each. Since $\Lambda_{j}^{l}(\forall j, l)$ is an $n_{t} \times n_{t}(=2 \times 2)$ matrix, it can be expressed as:

$$
\Lambda_{j}^{l}=\left[\begin{array}{cc}
\lambda_{j 1}^{l} & 0 \\
0 & \lambda_{j 2}^{l}
\end{array}\right],
$$

where $\lambda_{j 1}^{l}$ and $\lambda_{j 2}^{l}$ are the fractions of power transmitted by the $j^{\text {th }}$ user over the two Eigen-modes of the channel in the $l^{\text {th }}$ frequency band. To simplify the expressions for $x_{i}^{l}$ and $y_{i}^{l}$ (given by (7)), let us define $\widetilde{\mathbf{H}}_{j i}^{l}=\sqrt{\rho_{j i}^{l}} \widehat{\mathbf{H}}_{j i}^{l} . \mathbf{S}_{i}^{l}$ can now be expressed as (9). Taking the determinant of $\mathbf{S}_{i}^{l}, x_{i}^{l}$ can be expressed as (10). The expression for $y_{i}^{l}$ is similar to that for $x_{i}^{l}$ with the only difference being that the terms corresponding to $i^{t h}$ users are not included in the summations.

From (10) we observe that $x_{i}^{l}$ is a quadratic polynomial with three types of quadratic terms, viz., $\lambda_{j 1}^{l} \lambda_{k 1}^{l}, \lambda_{j 2}^{l} \lambda_{k 2}^{l}$ and $\lambda_{j 1}^{l} \lambda_{k 2}^{l}$. It is important to note that $\lambda_{j 1}^{l} \lambda_{k 1}^{l}$ is the same as $\lambda_{k 1}^{l} \lambda_{j 1}^{l}$ and hence the number of unique quadratic terms for this category is $\sum_{l=1}^{m} \sum_{j=1}^{N} j=\frac{m N}{2}(N+1)$. The same argument holds for $\lambda_{j 2}^{l} \lambda_{k 2}^{l}$. The number of unique terms for $\lambda_{j 1}^{l} \lambda_{k 2}^{l}(\forall j, k, l)$ is $m N^{2}$, which makes the total number of quadratic terms in (10) equal to $m N(2 N+1)$. Each of these quadratic terms is replaced by a new variable: $\Gamma_{j 1 k 1}^{l}=\lambda_{j 1}^{l} \lambda_{k 1}^{l}, \Gamma_{j 1 k 2}^{l}=\lambda_{j 1}^{l} \lambda_{k 2}^{l}$ and $\Gamma_{j 2 k 2}^{l}=\lambda_{j 2}^{l} \lambda_{k 2}^{l}$. These equalities are relaxed by including the following linear inequalities called bounding factor constraints (illustrated for $\Gamma_{j 1 k 2}^{l}$ ), for each non-linear term:

$$
\begin{aligned}
& \Gamma_{j 1 k 2}^{l}-\lambda_{j 1 L B}^{l} \lambda_{k 2}^{l}-\lambda_{j 1}^{l} \lambda_{k 2 L B}^{l} \geq-\lambda_{j 1 L B}^{l} \lambda_{k 2 L B}^{l} \\
& \Gamma_{j 1 k 2}^{l}-\lambda_{j 1 U B}^{l} \lambda_{k 2}^{l}-\lambda_{j 1}^{l} \lambda_{k 2 L B}^{l} \leq-\lambda_{j 1 U B}^{l} \lambda_{k 2 L B}^{l} \\
& \Gamma_{j 1 k 2}^{l}-\lambda_{j 1 L B}^{l} \lambda_{k 2}^{l}-\lambda_{j 1}^{l} \lambda_{k 2 U B}^{l} \leq-\lambda_{j 1 L B}^{l} \lambda_{k 2 U B}^{l} \\
& \Gamma_{j 1 k 2}^{l}-\lambda_{j 1 U B}^{l} \lambda_{k 2}^{l}-\lambda_{j 1}^{l} \lambda_{k 2 U B}^{l} \geq-\lambda_{j 1 U B}^{l} \lambda_{k 2 U B}^{l}
\end{aligned}
$$

This completes LPR construction of the original problem (3) 


$$
\begin{gathered}
\mathbf{S}_{i}^{l}=\left[\begin{array}{cc}
1+\sum_{j=1}^{N} \lambda_{j 1}^{l}\left(\tilde{h}_{j i}^{l}\right)_{11}\left(\tilde{h}_{j i}^{l}\right)_{11}^{\prime}+\lambda_{j 2}^{l}\left(\tilde{h}_{j i}^{l}\right)_{12}\left(\tilde{h}_{j i}^{l}\right)_{12}^{\prime} & \sum_{j=1}^{N} \lambda_{j 1}^{l}\left(\tilde{h}_{j i}^{l}\right)_{11}\left(\tilde{h}_{j i}^{l}\right)_{21}^{\prime}+\lambda_{j 2}^{l}\left(\tilde{h}_{j i}^{l}\right)_{12}\left(\tilde{h}_{j i}^{l}\right)_{22}^{\prime} \\
\sum_{j=1}^{N} \lambda_{j 1}^{l}\left(\tilde{h}_{j i}^{l}\right)_{21}\left(\tilde{h}_{j i}^{l}\right)_{11}^{\prime}+\lambda_{j 2}^{l}\left(\tilde{h}_{j i}^{l}\right)_{22}\left(\tilde{h}_{j i}^{l}\right)_{12}^{\prime} & 1+\sum_{j=1}^{N} \lambda_{j 1}^{l}\left(\tilde{h}_{j i}^{l}\right)_{21}\left(\tilde{h}_{j i}^{l}\right)_{21}^{\prime}+\lambda_{j 2}^{l}\left(\tilde{h}_{j i}^{l}\right)_{22}\left(\tilde{h}_{j i}^{l}\right)_{22}^{\prime}
\end{array}\right] \\
x_{i}^{l}=1+\sum_{j=1}^{N}\left(\left(\tilde{h}_{j i}^{l}\right)_{11}\left(\tilde{h}_{j i}^{l}\right)_{11}^{\prime}+\left(\tilde{h}_{j i}^{l}\right)_{21}\left(\tilde{h}_{j i}^{l}\right)_{21}^{\prime}\right) \lambda_{j 1}^{l}+\sum_{j=1}^{N}\left(\left(\tilde{h}_{j i}^{l}\right)_{12}\left(\tilde{h}_{j i}^{l}\right)_{12}^{\prime}+\left(\tilde{h}_{j i}^{l}\right)_{22}\left(\tilde{h}_{j i}^{l}\right)_{22}^{\prime}\right) \lambda_{j 2}^{l}+ \\
\sum_{j=1}^{N} \sum_{k=1}^{N}\left(\left(\tilde{h}_{j i}^{l}\right)_{11}\left(\tilde{h}_{j i}^{l}\right)_{11}^{\prime}\left(\tilde{h}_{k i}^{l}\right)_{21}\left(\tilde{h}_{k i}^{l}\right)_{21}^{\prime}-\left(\tilde{h}_{j i}^{l}\right)_{11}\left(\tilde{h}_{j i}^{l}\right)_{21}^{\prime}\left(\tilde{h}_{k i}^{l}\right)_{11}^{\prime}\left(\tilde{h}_{k i}^{l}\right)_{21}\right) \lambda_{j 1}^{l} \lambda_{k 1}^{l}+ \\
\sum_{j=1}^{N} \sum_{k=1}^{N}\left(\left(\tilde{h}_{j i}^{l}\right)_{12}\left(\tilde{h}_{j i}^{l}\right)_{12}^{\prime}\left(\tilde{h}_{k i}^{l}\right)_{22}\left(\tilde{h}_{k i}^{l}\right)_{22}^{\prime}-\left(\tilde{h}_{j i}^{l}\right)_{22}\left(\tilde{h}_{j i}^{l}\right)_{12}^{\prime}\left(\tilde{h}_{k i}^{l}\right)_{22}^{\prime}\left(\tilde{h}_{k i}^{l}\right)_{12}\right) \lambda_{j 2}^{l} \lambda_{k 2}^{l}+ \\
\sum_{j=1}^{N} \sum_{k=1}^{N}\left(\left(\tilde{h}_{j i}^{l}\right)_{11}\left(\tilde{h}_{j i}^{l}\right)_{11}^{\prime}\left(\tilde{h}_{k i}^{l}\right)_{22}\left(\tilde{h}_{k i}^{l}\right)_{22}^{\prime}+\left(\tilde{h}_{j i}^{l}\right)_{21}\left(\tilde{h}_{j i}^{l}\right)_{21}^{\prime}\left(\tilde{h}_{k i}^{l}\right)_{12}\left(\tilde{h}_{k i}^{l}\right)_{12}^{\prime}-\right. \\
\left.2 \Re\left(\left(\tilde{h}_{j i}^{l}\right)_{11}\left(\tilde{h}_{j i}^{l}\right)_{21}^{\prime}\right) \Re\left(\left(\tilde{h}_{j i}^{l}\right)_{12}\left(\tilde{h}_{j i}^{l}\right)_{22}^{\prime}\right)-2 \Im\left(\left(\tilde{h}_{j i}^{l}\right)_{11}\left(\tilde{h}_{j i}^{l}\right)_{21}^{\prime}\right) \Im\left(\left(\tilde{h}_{j i}^{l}\right)_{12}\left(\tilde{h}_{j i}^{l}\right)_{22}^{\prime}\right)\right) \lambda_{j 1}^{l} \lambda_{k 2}^{l}
\end{gathered}
$$

and the corresponding LP can be expressed as:

$$
\begin{array}{ll}
\max & \sum_{i=1}^{N} C_{i} \\
\text { s.t. } & C_{i}=\sum_{l=1}^{m} \frac{1}{\ln 2}\left(u_{i}^{l}-v_{i}^{l}\right),
\end{array}
$$

Polygonal approximation of $u_{i}^{l}$ and $v_{i}^{l}$ given by (6), Linear expressions for $x_{i}^{l}$ and $y_{i}^{l}$ (using (10)),

Bounding constraints for $\Gamma_{j 1 k 1}^{l}, \Gamma_{j 1 k 2}^{l}$ and $\Gamma_{j 2 k 2}^{l}$ (11), $\sum_{l=1}^{m} \operatorname{Tr}\left\{\Lambda_{i}^{l}\right\} \leq P_{\max }, \operatorname{diag}\left\{\Lambda_{i}^{l}\right\} \succeq \mathbf{0}$

$1 \leq i \leq N, 1 \leq l \leq m$.

The LPR essentially relaxes the feasible region of the original NL problem (3) and hence the optimal objective function value of the LP (12) serves as an UB to the optimal sum-rate. Since the LP is subject to the same power constraint as the original NL problem (3), the optimal solution to the LP is also a feasible solution to (3). In general, this feasible solution is not optimal to the original problem (3) and the corresponding objective function value of (3) yields a LB to the optimal sum-rate. BB strategy is then employed to partition the search space so that both the largest UB and LB approach towards the global maximum (as explained in [25], [26]).

\section{Numerical RESUlts}

The optimal sum-rate of cognitive MIMO IC is compared to two special cases, viz., no-interference (NI) and the IA bound, for different system parameters. The NI case is a hypothetical case and assumes that each SU is isolated from the other SUs in the network. This provides an upper bound to the optimal results. The IA bound represents the sum-rate achievable by performing IA. It is simply evaluated as half the sum-rate of the NI case. It should be noted that the IA bound is included just for the purpose of comparison and the feasibility of IA in limited signalling dimensions is still an open problem.

The channel model is assumed to be a combination of large scale and small scale fading components. On the large scale, we assume that channel suffers from an exponential path loss with a path loss exponent of 3 and from log-normal shadowing with a standard deviation of $1 \mathrm{~dB}$. Small scale fading effects are modeled as Rayleigh distributed. Antennas at both the Tx and $\mathrm{Rx}$ of all the users are assumed to be independent in terms of small-scale fading but perfectly correlated in terms of lognormal shadowing. The interference power is controlled by varying the $M U I$ factor (high $M U I$ means high interference power). $P_{\max }$ is set to one without loss of generality.

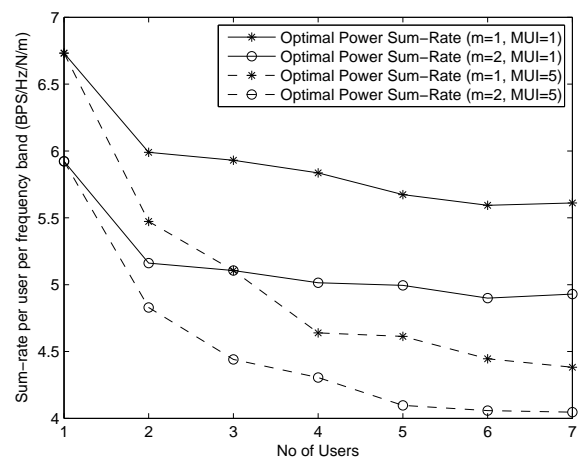

Fig. 3. Comparison of sum-rates of the single band and multiple band systems in low $(M U I=1)$ and high $(M U I=5)$ interference scenarios $\left(n_{t}=n_{r}=2\right)$.

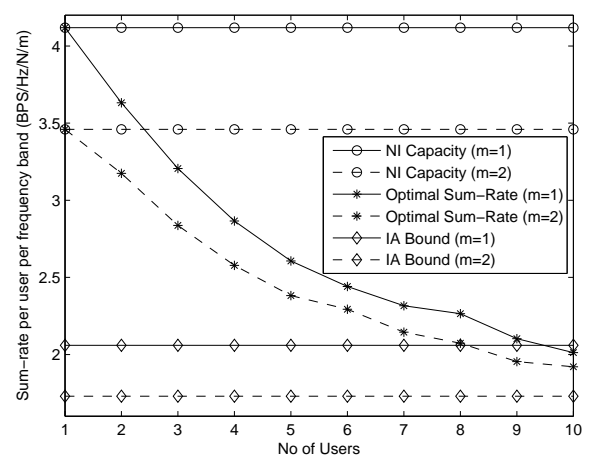

Fig. 4. Comparison of the sum-rates of single and multiple band systems for the case when $M U I=N\left(n_{t}=n_{r}=1\right)$.

In Fig. 3, we present the sum-rates achievable by the proposed optimal power allocation in low $(M U I=1)$ and high $(M U I=5)$ interference scenarios. As expected, the sumrate achievable in low interference scenario is higher than that achievable in the high interference scenario for both the single band and the multi-band MIMO IC. Another interesting observation is that the sum-rate scales better with the increase in frequency bands in the high interference scenario as compared to the low interference scenario. This means that the percentage increase in the sum-rate achieved by increasing the number of bands from one to two is higher in the high interference scenario as compared to the low interference scenario. This 


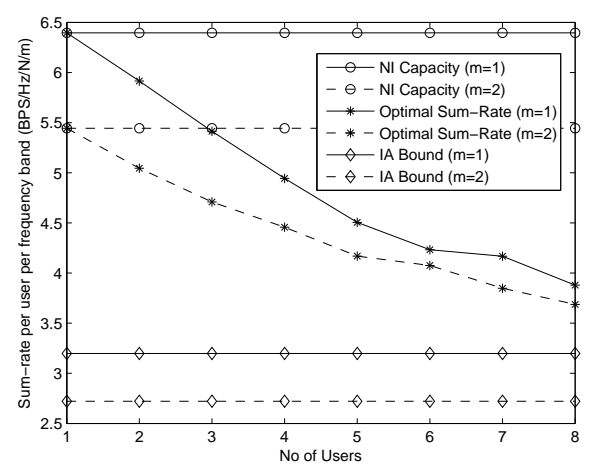

Fig. 5. Comparison of the sum-rates of single and multiple band systems for the case when $M U I=N\left(n_{t}=n_{r}=2\right)$.

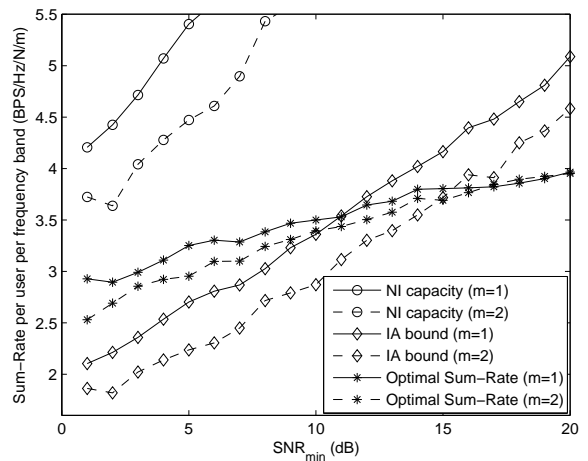

Fig. 6. Comparison of the sum-rates of single and multiple band systems at various values of $S N R_{\min }\left(M U I=N=4, n_{t}=n_{r}=1\right)$.

highlights the importance of interference avoidance in the high interference scenarios. We also note that the rate of decrease of sum-rate reduces considerably for $N>M U I$. This is because the number of dominant interferers (lying inside the circle of radius $d_{\max }$ ) is constant for $N>M U I$, whereas, the less dominant interferers (lying outside the circle) continues to grow with $N$. It should also be noted that IA is most useful in the cases when all the users interfere strongly with each other. Therefore, we consider $M U I=N$ in all our subsequent cases to facilitate a fair comparison between the optimal power sumrate and the IA bound.

In Fig. 4, we consider a SISO IC and compare the optimal sum-rate to the NI capacity and the IA bound. The first observation is that the NI capacity per frequency band reduces when we increase $m$ from 1 to 2 . This is due to the fact that doubling the number of bands does not allow a doubling of capacity, thus the capacity per frequency band goes down. The optimal sum-rate per user per frequency band is observed to be higher than the IA bound for a small to moderate number of SUs. The per user sum-rate decreases with the increase in interference power in the optimal power control case but remains constant in the IA case. Due to this, IA starts performing better than the proposed optimal method after a certain cross-over point (referred henceforth as $N_{C O}$ ). This highlights the effectiveness of IA in high interference scenarios. Another interesting observation is that the cross-

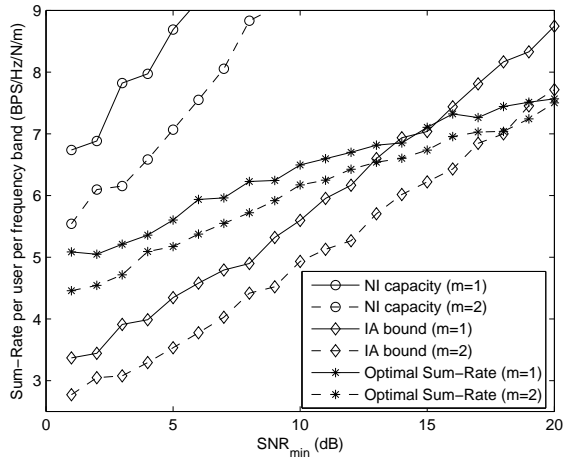

Fig. 7. Comparison of the sum-rates of single and multiple band systems at various values of $S N R_{\min }\left(M U I=N=4, n_{t}=n_{r}=2\right)$.

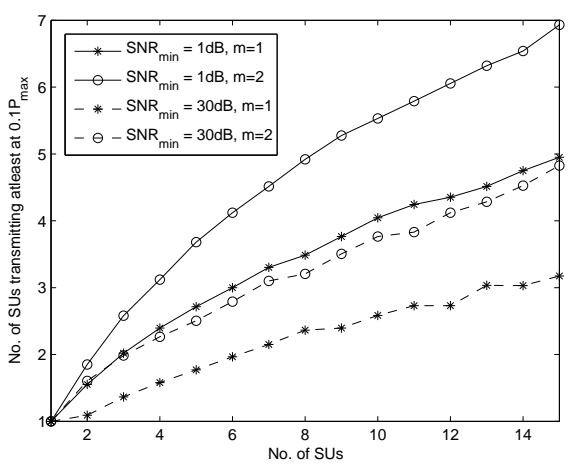

Fig. 8. The number of SUs allowed to transmit in the SISO IC for $S N R_{\text {min }}=1 d B$ and $S N R_{\text {min }}=30 \mathrm{~dB}\left(N_{t}=N_{r}=1, M U I=N\right)$.

over point $N_{C O}$ is higher when there are a higher number of orthogonal frequency bands available for transmission. This means that the performance of the proposed technique improves significantly with the increase in the number of orthogonal frequency bands. This is an important result from the CR perspective, where we expect to have several orthogonal frequency bands for the use of SUs. A similar comparison is carried out for a $2 \times 2$ cognitive MIMO IC in Fig. 5. In addition to the already mentioned results, we observe that the performance of the optimal power control technique is better in this case than the SISO IC case due to the presence of orthogonal spatial channels. In particular, the cross-over points $N_{C O}$ are higher in this case than their counterparts in the SISO IC case. Thus, the presence of orthogonal spatial and/or spectral channels improves the interference avoidance capability of the power control technique. We also note that the sum-rate scales better with the increase in the number of orthogonal frequency bands in the high interference case.

In Fig. 6, we study the effect of $S N R_{\min }$ on the performance of our proposed technique and its comparison to the IA bound. We consider a four-user SISO IC and compare the optimal results with the NI capacity and the IA bound. We observe that the proposed power control technique performs better than the IA bound in the low $S N R$ regime. This is due to the fact that the sum-rates achievable in IA are highly suboptimal in the low $S N R$ regime. As we increase $S N R_{\text {min }}$, 
IA starts performing better than the proposed technique after a certain $S N R_{\min }$ value (referred henceforth as $S N R_{C O}$ ). We also observe that the $S N R_{C O}$ is higher when there are multiple bands for transmission. This reiterates the fact that the performance of the optimal power control technique improves as compared to IA bound when there are more orthogonal frequency bands available for transmission. Repeating the same comparison for the $2 \times 2$ cognitive MIMO IC in Fig. 7, we observe that the cross-over points, $S N R_{C O}$, are higher in this case as compared to the SISO case. Another interesting observation from Fig. 6 and 7 is that the sum-rate scales better with the increase in the number of orthogonal frequency bands for the optimal power control case in the high $S N R$ regime. To explain this behavior, we briefly look at the fairness of proposed power allocation scheme in the SISO IC in Fig. 8. In particular, we calculate the number of SUs that are allowed to transmit at least $10 \%$ of $P_{\max }$ in the SISO IC. We first observe that fewer SUs are allowed to transmit in the high $S N R$ regime as compared to the low $S N R$ regime. This is due to the fact that the interference links are stronger in the high $S N R$ regime (relative to the noise power) and it is optimal to turn-off more SUs to maximize the sum-rate as compared to the low SNR regime (analogous to results presented in [27] for symmetric ICs). More importantly, we note that the percentage increase in the number of 'transmitting' SUs by adding an extra frequency band is higher in the high $S N R$ regime. For example, the percentage increase in the number of 'transmitting' SUs (for 4-user SISO IC) is $45 \%$ in the $30 \mathrm{~dB} S N R_{\min }$ case as opposed to $30 \%$ in the $1 \mathrm{~dB} S N R_{\min }$ case. A larger increase in the number of 'transmitting' SUs leads to a better capacity scaling in the high $S N R$ regime. It should be noted that the fairness of resource allocation in the proposed technique requires further investigation and will be discussed in a separate publication.

\section{ACKNOWLEDGMENTS}

We would like to thank Qualcomm Inc. for sponsoring this research. Thanks are due to Dr. Thomas Hou and Kevin Liu for their help in understanding the BB/RLT algorithm.

\section{CONCLUSION}

In this paper, we have developed a global optimization algorithm to find the maximum sum-rate achievable by employing spatio-spectral power control in the cognitive MIMO IC treating interference as Gaussian noise. We have shown that this spatio-spectral power control yields higher sum-rate than that of IA in low interference and low $S N R$ scenarios. The performance of the proposed power control technique improves with an increase in the number of orthogonal frequency and/or spatial channels. These results are promising from the CR perspective, where SUs typically operate in a relatively low $S N R$ regime and are generally expected to have multiple frequency bands available for transmission. We have also shown that the optimal power control technique achieves better sum-rate scaling (with an increasing number of orthogonal frequency bands) in the high interference and high $S N R$ scenarios.

\section{REFERENCES}

[1] J. Mitola, "Cognitive Radio for Flexible Mobile Multimedia Communication," in Proc. IEEE Int. Workshop Mobile Multimedia Comm. $($ MoMиC), San Diego, CA, Nov. 1999, pp. 3-10.

[2] J. Mitola, "Cognitive Radio: An Integrated Agent Architecture for Software Defined Radio", Ph.D. Dissertation, KTH, 2000.

[3] S. Haykin, "Cognitive Radio: Brain-Empowered Wireless Communications", IEEE J. Select. Areas Comm., vol. 23, no. 2, pp. 201-220, 2005.

[4] I. F. Akyildiz, W. -Y. Lee, M. C. Vuran and S. Mohanty, "NeXt Generation/Dynamic Spectrum Access/Cognitive Radio Wireless Networks: A Survey", Comput. Networks, vol. 50, no. 13, pp. 2127-2159, 2006.

[5] FCC Spectrum Policy Task Force, "FCC Report of the Spectrum Efficiency Working Group", Nov. 2002.

[6] Q. Zhao and B. Sadler, "A Survey of Dynamic Spectrum Access", IEEE Sig. Process. Mag., vol. 24, no. 3, pp. 79-89, May 2007.

[7] Y. Xing, R. Chanddramouli, S. Mangold and S. Shankar, "Dynamic Spectrum Access in Open Spectrum Wireless Networks", IEEE J. Sel. Areas Commun., vol. 24, pp. 626-636, Mar. 2006.

[8] W. Lehr and J. Crowcroft, "Managing Shared Access to a Spectrum Commons", in Proc. IEEE DySPAN, Nov. 2005, pp. 420-444.

[9] S. Shankar, C. Cordeiro and K. Challapali, "Spectrum Agile Radios: Utilization and Sensing Architectures", in Proc. IEEE DySPAN, Baltimore, MD, Nov. 2005, pp. 160-169.

[10] C. E. Shannon, "Two-Way Communication Channels", in Proc. $4^{\text {th }}$ Berkeley Symp. Math. Stat. Prob., Berkeley, CA, 1961, pp. 611-644.

[11] R. Ahlswede, "The Capacity Region of a Channel with Two Senders and Two Receivers", Annals Probability, vol. 2, no. 5, pp. 805-814, 1974.

[12] A. B. Carleial, "Interference Channels", IEEE Trans. Inform. Theory, vol. IT-24, pp. 60-70, Jan. 1978.

[13] H. Sato, "Two-User Communication Channels", IEEE Trans. Inform. Theory, vol. IT-23, pp. 295-304, May 1977.

[14] A. B. Carleial, "A Case where Intereference does not Reduce Capacity", IEEE Trans. Inform. Theory, vol. IT-21, pp. 569-570, Sept. 1975.

[15] T. S. Han and K. Kobayashi, "A New Achievable Rate Region for the Interference Channel", IEEE Trans. Inform Theory, vol. 27, no. 1, pp. 49-60, Jan. 1981.

[16] R. Etkin, D. Tse and H. Wang, "Gaussian Interference Channel Capacity to Within One Bit", IEEE Trans. Inform. Theory, vol. 54, no. 12, pp. 5534-5562, 2008.

[17] M. Maddah-Ali, A. Motahari and A. Khandani, "Signaling over MIMO Multi-Base systems- Combination of Multi-Access and Broadcast Schemes", in Proc. IEEE Int. Symp. Inform. Theory, Seattle, WA, 2006.

[18] V. R. Cadambe and S. A. Jafar, "Interference Alignment and the Degrees of freedom for the $K$ user interference channel", IEEE Trans. Inform. Theory, vol. IT-54, no. 8, pp. 3425-3441, Aug. 2008.

[19] M. Shen, A. H $\phi$ st-Madsen and J. Vidal, "An Improved Interference Alignment Scheme for Frequency Selective Channels", in Proc. IEEE Int. Symp. on Inform. Theory, July 2008, pp. 559-563.

[20] S. W. Choi, S. A. Jafar and S.-Y. Chung, "On the Beamforming Design for Efficient Interference Alignment", IEEE Comm. Lett., vol. 13, no. 11, pp. 847-849, 2009.

[21] T. Gou and S. A. Jafar, "Degrees of Freedom of the K User MIMO Interference Channel", in Proc. Asilomar Conf. Sig., Sys. and Computers, Pacific Grove, CA, 2008, pp. 126-130.

[22] R. Tresch, M. Guillaud and E. Riegler, "On the Achievability of Interference Alignment in the K-user Constant MIMO Interference Channel", in Proc. IEEE Workshop on Statistical Signal Processing, Cardiff, U.K., Sept. 2009, pp. 277-280.

[23] H. S. Dhillon and R. M. Buehrer, "Cognitive MIMO Radio: Incorporating Dynamic Spectrum Access in Multiuser MIMO Network", in Proc. IEEE GLOBECOM, Honolulu, HI, Nov. 30 - Dec. 5, 2009.

[24] B. S. Blum, "MIMO Capacity with Interference", IEEE J. Select. Areas Commun., vol. 21, pp. 793-801, June 2003.

[25] J. Liu, Y. T. Hou, Y. Shi and H. Sherali, "On the Capacity of Multiuser MIMO Networks with Interference", IEEE Trans. Wireless Commun., vol. 7, no. 2, pp. 488-494, Feb. 2008.

[26] H. S. Dhillon and R. M. Buehrer, "On the Sum-Rate of MIMO Interference Channel", accepted for publication in IEEE GLOBECOM 2010.

[27] S. R. Bhaskaran, S. V. Hanly, N. Badruddin, J. S. Evans, "Maximizing the Sum Rate in Symmetric Networks of Interfering Links", in Proc. IEEE ICC, Dresden, June 2009. 